Proceedings

\title{
Detecting Anomalous Noise Events on Low-Capacity Acoustic Sensor in Dynamic Road Traffic Noise Mapping ${ }^{\dagger}$
}

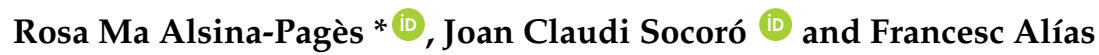 \\ GTM-Grup de recerca en Tecnologies Mèdia, La Salle-Universitat Ramon Llull, Quatre Camins, 30, \\ 08022 Barcelona, Spain; jclaudi@salleurl.edu (J.C.S.); falias@salleurl.edu (F.A.) \\ * Correspondence: ralsina@salleurl.edu; Tel.: +34-93-2902455 \\ t Presented at the 4th International Electronic Conference on Sensors and Applications, 15-30 November 2017; \\ Available online: https://sciforum.net/conference/ecsa-4.
}

Published: 14 November 2017

\begin{abstract}
One of the main aspects affecting the life of people living in urban and suburban areas is their continued exposure to high road traffic noise (RTN) levels, traditionally measured by specialists working on the field. Nowadays, the deployment of Wireless Acoustic Sensor Networks (WASN) has allowed to automate noise mapping in Smart Cities. In order to obtain a reliable picture of the RTN levels affecting citizens, those anomalous noise events (ANE) unrelated to road traffic should be removed from the noise map computation. For this purpose, an Anomalous Noise Event Detector (ANED) designed to differentiate in real-time between RTN and ANE should be developed to run on the low-cost acoustic sensors of the WASN. In this work, the viability of implementing the ANED algorithm to run on low-capacity (LowCap) $\mu$ controller-based acoustic sensors developed within the DYNAMAP project is presented, after being designed and implemented for the high-capacity sensors. The algorithm is based on the comparison between RTN and ANE spectral differences using real-life acoustic data from both suburban and urban scenarios. The results show significant spectral differences between RTN and ANE classes in both environments, after being parametrized using Gammatone Cepstral Coefficients. However, further research should be conducted to determine the most discriminant subbands, which should be taken into account for the implementation of the ANED LowCap version.
\end{abstract}

Keywords: low capacity; low cost; anomalous event; algorithm; noise; real-time detection; road traffic noise; background noise; dynamic noise mapping

\section{Introduction}

Living with continuous exposure to high levels of traffic noise has been proved to be harmful for health, affecting the quality of life of people living in urban and suburban areas [1]. Several actions have been conducted to address this problem, taking account of the European Noise Directive 2002/49/EC (END) [2] and the consequent strategic noise mapping assessment CNOSSOS-EU [3], which are the main instruments at the European level devoted to elaborate specific action plans to mitigate noise pollution.

Noise measurements in cities have been historically conducted by professionals, recording and analyzing the data in specific locations and time periods by using certified sound level meters. The noise maps are then generated from these noise level measurements by means of the application of complex acoustic models after data post-processing; maps that should be updated and published every five years to fulfill the END requirements [2]. However, this approach becomes difficult to scale when more measurements and/or locations are needed. The Internet of Things and the Smart 
City frameworks have allowed a change of paradigm for the city noise management and policy generation by means of the deployment of Wireless Acoustic Sensor Networks (WASN) [4]. The core idea of the development of these networks is designing and deploying low-cost reliable and scalable automatic sensing system. Several projects based on WASN have been developed in Europe, such as the RUMEUR network [5] in France, which actually is an hybrid network: it includes both high accuracy equipment for critical places, like airports, where the focus is to obtain detailed acoustic information due to the intense noise environment, together with less precise measuring equipment in other locations where the goal is only the updating of the noise map. Other projects are the IDEA project [6] in Belgium, or the SoNYC project [7] in New York City, which provides a description of the acoustic environment of the city.

Recently, the DYNAMAP project aims at developing a dynamic noise mapping system to represent the acoustic impact of the road traffic noise in real time in two Italian pilot areas, Rome and Milan [8]. The WASN envisioned for the DYNAMAP project is hybrid, composed of both low-cost high-capacity sensors and low-cost low-computation capacity sensors, as in RUMEUR network [5]. The DYNAMAP project takes into account an acoustic measurement challenge not faced by the previous project as it considers the inclusion of an Anomalous Noise Event Detection algorithm [9] to provide a reliable picture of actual road traffic noise (RTN). Moreover, anomalous noise events (e.g., aircrafts, trains, bells) should be automatically identified and removed in order to cope with the noise source distinction required by the END [2]. In order to remove this kind of events from the RTN measurement of the WASN, the ANED algorithm has been designed as a two-class classifier (ANE vs. RTN) [9], whose outputs are obtained following a two-level decision process; the frame-level binary decisions are integrated through a high-level decision based on a majority voting scheme computed in a certain interval. The algorithm has been developed and tested in the two pilot areas of the DYNAMAP project-the reader can find more information about the algorithm details and performance evaluation in [9].

The ANED algorithm has been initially designed and developed to run on the high-capacity sensors taking advantage of their computing power. In this paper, the preliminary analysis of the viability to adapt the ANED to the low-capacity sensors (denoted as ANED LowCap) is presented. The core idea of the ANED LowCap proposal is to reduce its computational complexity by means of proposing a simplified classification scheme based on a signal threshold obtained directly from the A-weighted band energy filtering process of the raw acoustic signal. In this small piece of research we present the first analysis conducted over two recorded and labelled datasets in Milan and in Rome [10], to test the viability of the band energy study as a basic binary classifier using a threshold.

This paper is structured as follows. In Section 2, the proposal to adapt the ANED algorithm to run on the low-cost low-capacity acoustic sensors is presented. In Section 3, we describe the analysis of viability of the approach over raw acoustic data collected from both suburban and urban scenarios of operation. In Section 4, we discuss the open questions foreseen from the results obtained in the previous section, which should be tackled in future works in order to implement the ANED LowCap.

\section{ANED LowCap Proposal}

The ANED LowCap algorithm proposal is mainly focused on the reduction of the computational complexity of the ANED algorithm designed to run on the high-capacity sensors [9] while maintaining its capability to identify and discard ANE from the RTN noise level computation. In order to implement the algorithm in an affordable low capacity computational platform with a microcontroller as a core, a change of paradigm is mandatory, both in terms of feature extraction and classification, so as to satisfy the severe decrease of the computational cost.

In Figure 1, the block diagram of the ANED version of adapted to run on the low computation capacity sensors is depicted. The signal level computation block obtains a variable that is directly related to the signal acoustic level computed per several spectral bands, by including a band-pass filtering step. Then, the classification process consists of a simple comparison between the signal level measure and the optimized threshold $\gamma$. With the aim of obtaining reliable output estimation of the 
RTN and ANE labels, the threshold $\gamma$ should be calculated in an optimization process based on audio databases analysis.

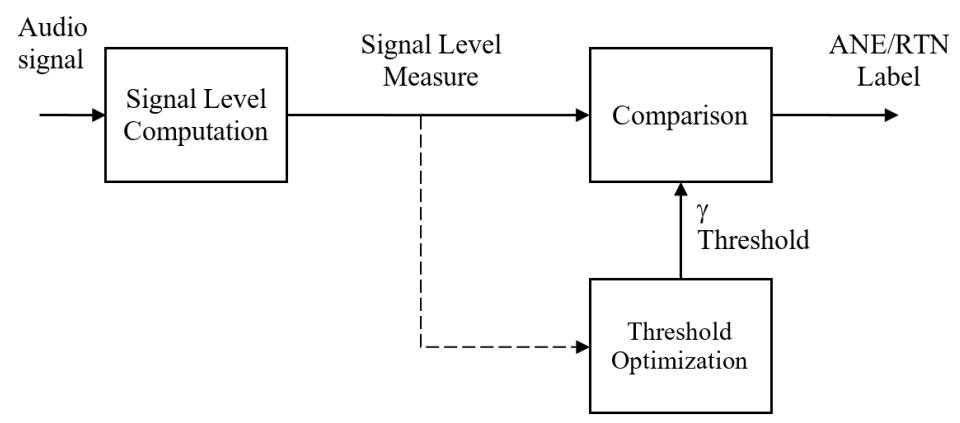

Figure 1. Block diagram of the ANED LowCap version adapted to run on low-cost low-capacity acoustic sensors.

\section{Analysis}

The analysis of the viability of the aforementioned approach is based on the computation of the spectral envelopes of the recorded audio signals, windowed at $30 \mathrm{~ms}$, and its subsequent clustering. The purpose of this cluster analysis is to evaluate to what extent there are significant spectral differences between RTN and ANE for each database (Rome and Milan) at certain spectral bands, from real-life collected acoustic data.

Two types of analysis have been conducted after the parametrization of the computed spectral envelopes of each database using Gammatone Cepstral Coefficients (GTCC) [11]:

- $\quad$ RTN analysis: we have performed an automatic clustering using Gaussian Mixture Models (GMM), sweeping the number of clusters from 1 to 6 to observe differences.

- ANE analysis: we have performed a supervised clustering based on subcategories defined during the database labelling process [12]. Accordingly, we have grouped the results into ANE categories, containing the available data per category for each dataset.

\subsection{RTN Spectral Analysis}

Figure 2 shows the clustering analysis of the RTN class in Rome for 1, 2, 4 and 6 clusters. The idea of testing with several clusters in the RTN class comes from the fact that there are several types of traffic noise, depending on the sound source. The ANE class has labelled each different type of sound depending on the source (e.g., people, siren, thunder), but in the RNT this labelling process would be diffuse, because sometimes several types of traffic occur together. Analyzing with different types of clusters for RTN will give information about the diversification of the traffic noise.

In the following figures, the mean frequencies are represented with solid lines while the mean plus and minus the standard deviation are depicted using dashed lines. Figure 3, depicts the same information for the city of Milan.

It is worth noticing that RTN exhibits different patterns for the city of Rome (suburban environment) and the city of Milan (urban environment). Moreover, both Figures 2 and 3 show that an increase in the number of GMM components makes the results to better reflect the different intensity profiles present in the RTN measurements. In this sense, in the suburban environment of the Rome ring, more homogeneous profiles are observed than in the urban context of the Milan city, as they contain RTN coming from more regular traffic conditions as the sensors are placed on the portals of the Rome's A90 motorway. 

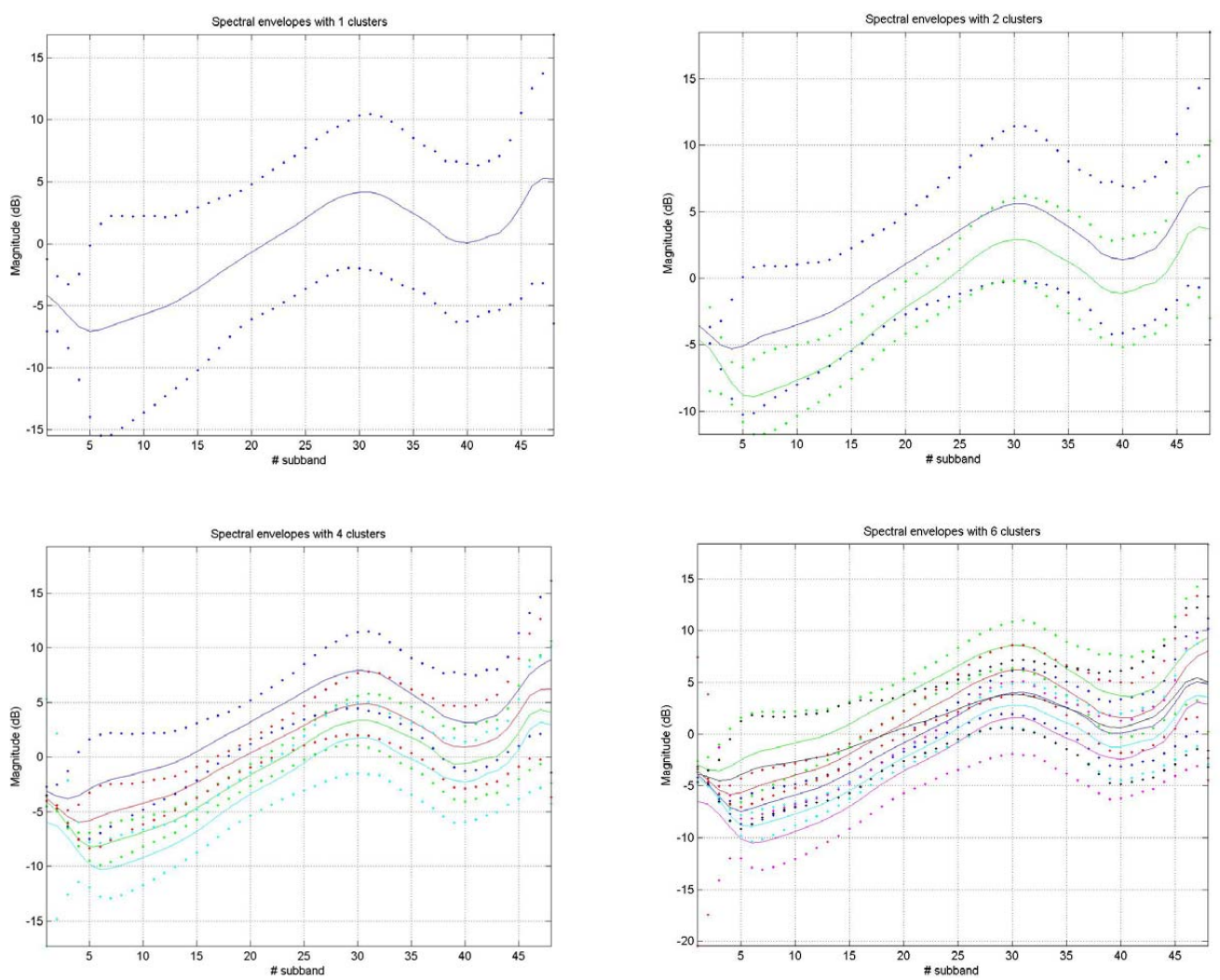

Figure 2. Clustering analysis at the spectral level of the RTN class in the Rome recordings.
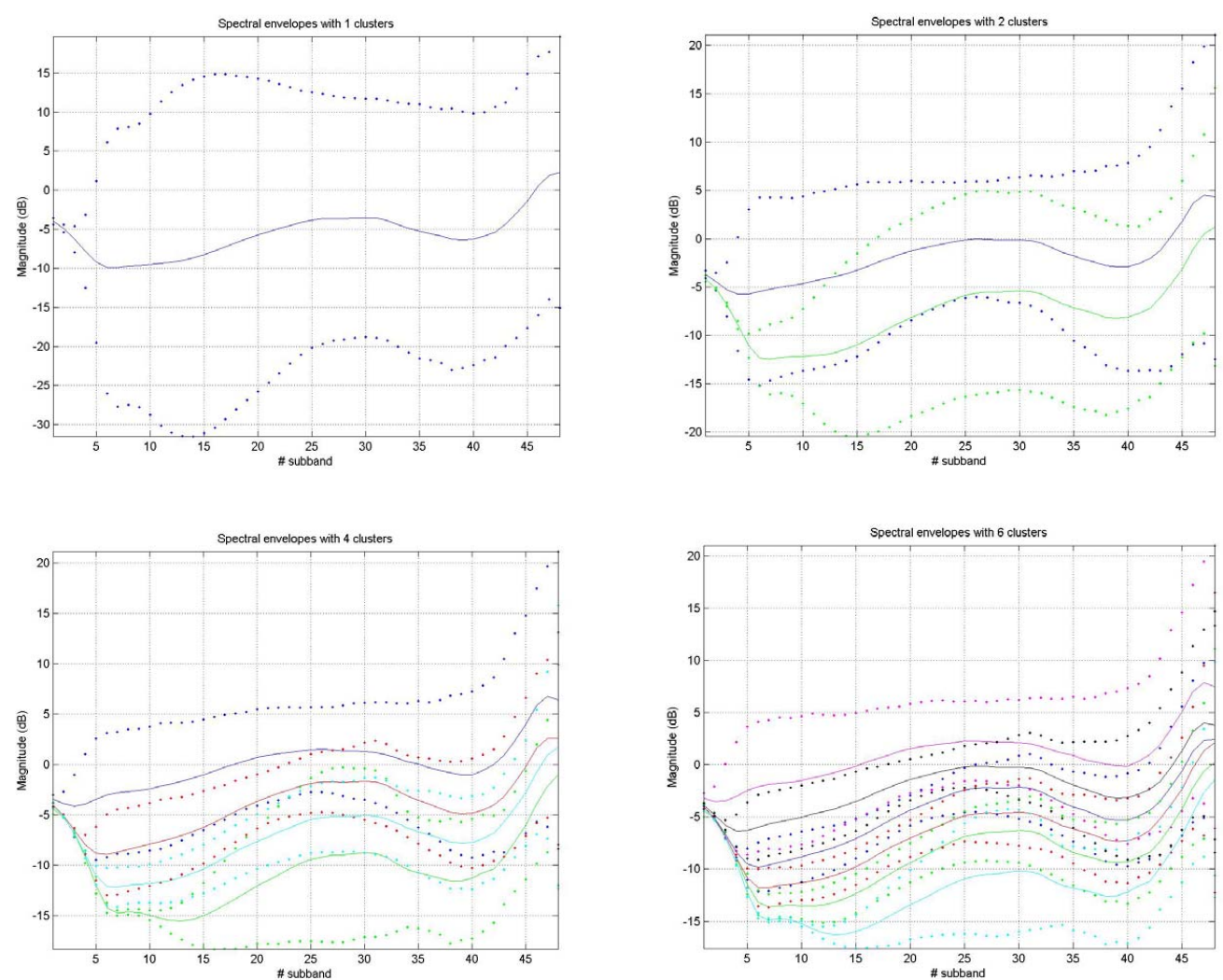

Figure 3. Clustering analysis at the spectral level of the RTN class in the Milan recordings. 


\subsection{ANE Spectral Analysis}

In Figures 4 and 5, the cluster analysis of the ANE class is depicted for both Rome and Milan cities. The depicted ANE are different in the suburban and urban scenarios. Again, the mean frequency is drawn using solid lines and the mean plus and minus the standard deviation is plotted using dashed lines.
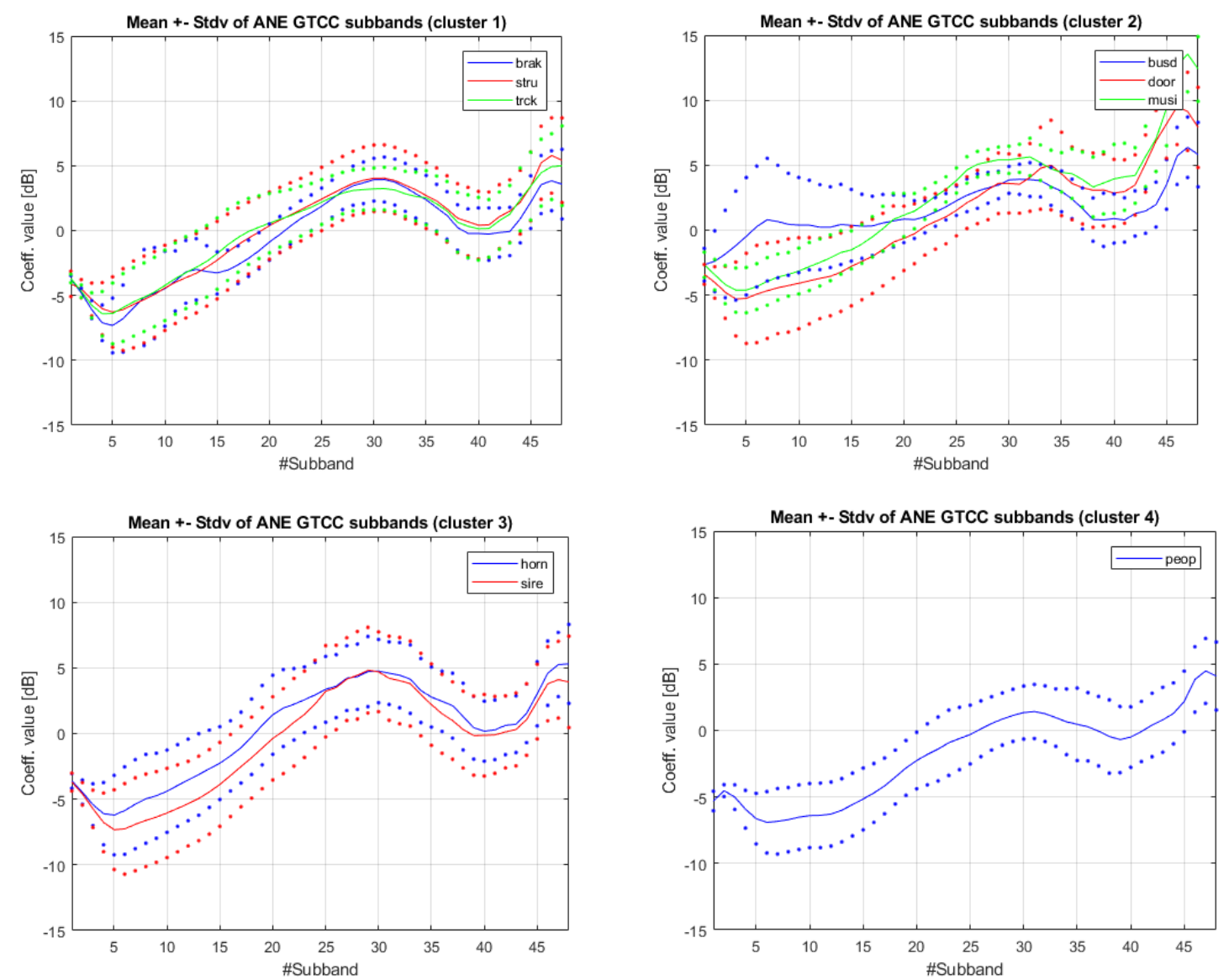

Figure 4. Clustering analysis at spectral level of the ANE class in the Rome recordings.

From these results, on the one hand, we can observe that ANE and RTN exhibit similar patterns for each city database. On the other hand, subtle but not negligible differences can be observed when comparing statistics of the clustered spectral envelopes of the RTN and ANE classes across the 48 frequency bands.

Therefore, it can be concluded that the differences found between RTN and ANE for each scenario can be of use for the implementation of the ANED LowCap. Nevertheless, the specific study of which frequency subbands should be considered in the signal level module of Figure 1 capable of providing the best discrimination capabilities is a matter of research left for future work. 

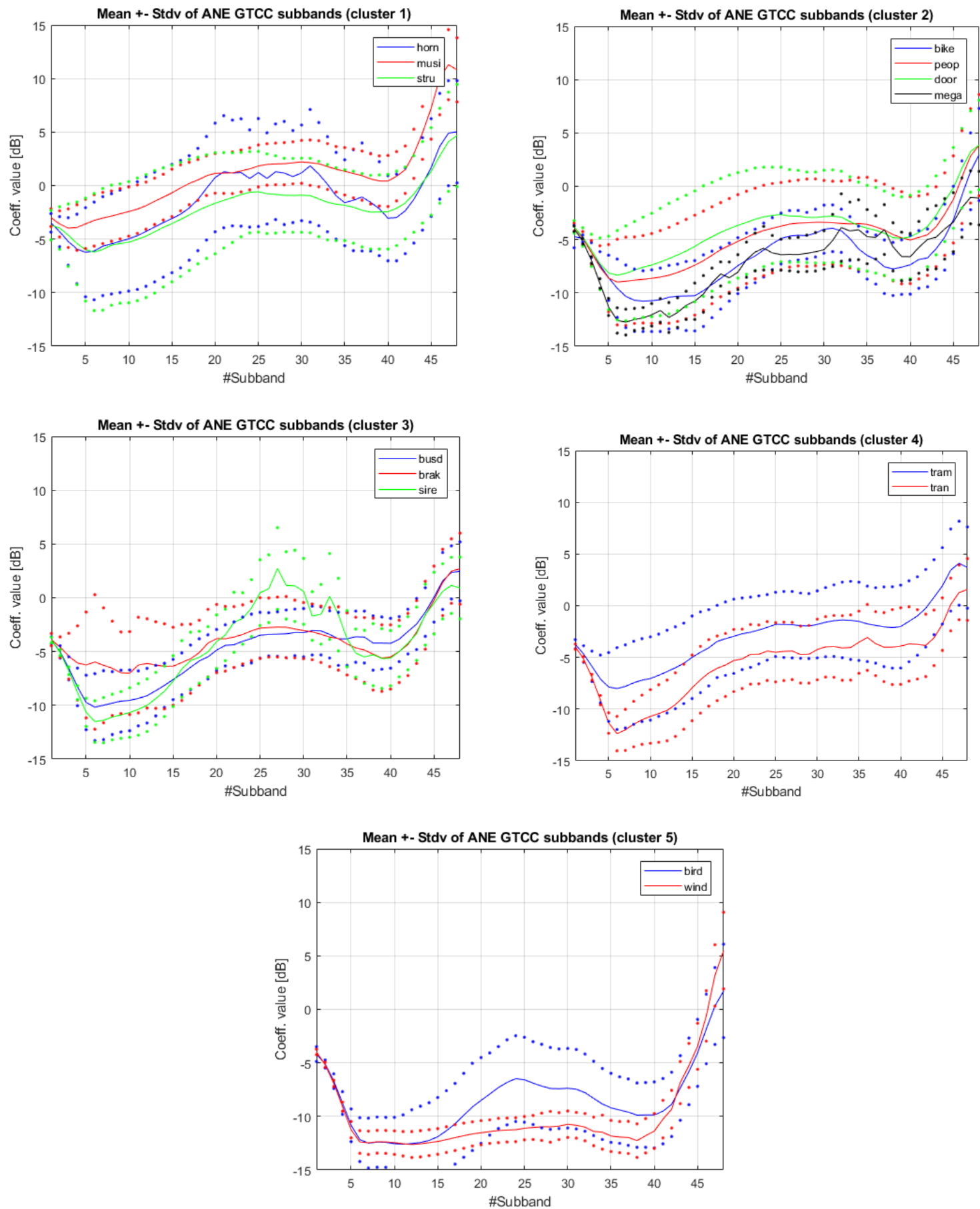

Figure 5. Clustering analysis at spectral level of the ANE class in the Milan recordings.

\section{Discussion}

The results of the spectral analysis conducted over real-life acoustic data collected from both suburban and urban environments show significant differences between the RTN and the ANE spectral distributions. Thus, it seems viable to take into account these differences in order to implement the ANED LowCap version for low-cost low-capacity acoustic sensors. However, it is difficult to determine which are the GTCC bands where spectral differences between RTN and ANE occur, and hence, should be selected to derive a simple yet effective threshold-based binary classifier (i.e., a low-cost solution with higher reliability than using only LAeq computed from the wide-band audio signal spectrum). 
For this reason, an in-depth quantitative analysis based on the identification of the frequency bands yielding lower error probability rates is proposed to be the next step to design the ANED LowCap algorithm version.

The future analysis of the ANED LowCap is devised following the next steps: (i) first, a preliminary approach based on a simple thresholding considering the LAeq measures over the full-band signal can be firstly explored as a means of obtaining a baseline system with which to compare; (ii) second, the classification threshold should be adjusted to differentiate between ANE and RTN categories optimally for each frequency band of interest to define the decision rules of the low-capacity ANED; (iii) and finally, a validation of the algorithm is foreseen to assess the performance of the design in suburban and urban scenarios in comparison with the ANED version already developed for the low-cost high-capacity acoustic sensors composing the hybrid WASN of the DYNAMAP project.

Acknowledgments: This research has been partially funded by the European Commission under project LIFE DYNAMAP LIFE13 ENV/IT/001254 and the Secretaria d'Universitats i Recerca del Departament d'Economia i Coneixement (Generalitat de Catalunya) under grant ref. 2014-SGR-0590 and grant ref. 2017-URL-Proj-013.

Author Contributions: Rosa Ma Alsina-Pagès wrote the paper. Joan Claudi Socoró performed the tests and reviewed the paper. Francesc Alías contributed to the writing of the paper and helped in the design of the experiments.

Conflicts of Interest: The authors declare no conflict of interest.

\section{Abbreviations}

The following abbreviations are used in this manuscript:

$\begin{array}{ll}\text { ANED } & \text { Anomalous Noise Event Detection } \\ \text { CPU } & \text { Central Processing Unit } \\ \text { EC } & \text { European Commission } \\ \text { END } & \text { Environmental Noise Directive } \\ \text { EU } & \text { European Union } \\ \text { GTCC } & \text { Gammatone Cepstrum Coefficients } \\ \text { MFCC } & \text { Mel-Frequency Cepstrum Coefficients } \\ \text { WASN } & \text { Wireless Acoustic Sensor Network }\end{array}$

\section{References}

1. Babisch, W. Transportation noise and cardiovascular risk. Noise Health 2008, 10, 27-33.

2. E.U. EU Directive: Directive 2002/49/EC of the European Parliament and the Council of 25 June 2002 relating to the assessment and management of environmental noise. Off. J. Eur. Commun. 2002, L 189/12.

3. Kephalopoulos, S.; Paviotti, M.; Ledee, F.A. Common Noise Assessment Methods in Europe (CNOSSOS-EU); Publications Office of the European Union: Rue Mercier, Luxembourg, 2012.

4. Bertrand, A. Applications and trends in wireless acoustic sensor networks: A signal processing perspective. In Proceedings of the 18th IEEE Symposium on Communications and Vehicular Technology in the Benelux (SCVT), Ghent, Belgium, 22-23 November 2011; pp. 1-6.

5. Mietlicki, F.; Mietlicki, C.; Sineau, M. An innovative approach for long-term environmental noise measurement: RUMEUR network. In Proceedings of the EuroNoise 2015, Maastrich, The Netherlands, 31 May-3 June 2015; pp. 2309-2314.

6. Botteldooren, D.; De Coensel, B.; Oldoni, D.; Van Renterghem, T.; Dauwe, S. Sound monitoring networks new style. In Proceedings of the Acoustics 2011: Breaking New Ground: Annual Conference of the Australian Acoustical Society, Australian Acoustical Society, Gold Coast, Australia, 2-4 November 2011; pp. 1-5.

7. SonYC-Sounds of New York City. Available online: https://wp.nyu.edu/sonyc (accessed on 14 September 2017).

8. Sevillano, X.; Socoró, J.C.; Alías, F.; Bellucci, P.; Peruzzi, L.; Radaelli, S.; Coppi, P.; Nencini, L.; Cerniglia, A.; Bisceglie, A.; Benocci, R.; Zambon, G. DYNAMAP—Development of low cost sensors networks for real time noise mapping. Noise Mapp. 2016, 3, 172-189. 
9. Socoró, J.C.; Alías, F.; Alsina-Pagès, R.M. An Anomalous Noise Events Detector for Dynamic Road Traffic Noise Mapping in Real-Life Urban and Suburban Environments. Sensors 2017, 17, 2323.

10. Alías, F.; Alsina-Pagès, R.M.; Socoró, J.C.; Orga, F.; Nencini, L. Performance analysis of the low-cost acoustic sensors developed for the DYNAMAP project: A case study in the Milan urban area. J. Acoust. Soc. Am. 2017, 141, 3883-3884.

11. Valero, X.; Alías, F. Gammatone Cepstral Coefficients: Biologically Inspired Features for Non-Speech Audio Classification. IEEE Trans. Multimed. 2012, 14, 1684-1689.

12. Alías, F.; Socoró, J.C. Description of Anomalous Noise Events for Reliable Dynamic Traffic Noise Mapping in Real-Life Urban and Suburban Soundscapes. Appl. Sci. 2017, 7, 146.

(C) 2018 by the authors. Licensee MDPI, Basel, Switzerland. This article is an open access article distributed under the terms and conditions of the Creative Commons Attribution (CC BY) license (http://creativecommons.org/licenses/by/4.0/). 